\title{
A Stepwise Approach to Identify the Clinical Role of FDG PET-CT in Patients with Suspicious Bone Metastasis from an Unknown Primary Site
}

\author{
Md. Sunny Anam Chowdhury'1, Subin Jeon'2, Zeenat Jabin², Hee-Seung Bom², Jung-Joon Min², Seong Young Kwon²
}

\author{
1 Institute of Nuclear Medicine \& Allied Sciences, Bogura, Bangladesh \\ 2 Chonnam National University Hwasun Hospital, Republic of Korea \\ 3 National Institute of Nuclear Medicine \& Allied Sciences, Dhaka, Bangladesh
}

Correspondence Address: : Dr. Md. Sunny Anam Chowdhury, Institute of Nuclear Medicine \& Allied Sciences, Shaheed Ziaur Rahman Medical College Hospital, Bogura, G.P.O Box \# 61, Bogura - 5800 Email: drsunny43@gmail.com

\begin{abstract}
Objectives: In patients with suspicious bone metastasis, differential diagnosis and primary lesion identification is very important in optimizing treatment planning. The study was aimed to assign a step-wise approach based on image findings to identify the precise role of PET-CT in these patients.
\end{abstract}

Patients and Methods: A total of 74 patients were enrolled with suspicious bone metastasis who underwent PET-CT for the evaluation of primary focus. Patients were classified into four groups with stepwise manners, firstly based on FDG positivity of bone lesions, then on multiplicity of positive bone uptake and finally on presence of extra-skeletal uptake. We analyzed the difference of distribution of follow-up results according to each group. In addition, we also investigated whether PET-CT had a role to guide biopsy sites.

Results: Except for 7 patients without bone or extra-skeletal uptake, 44 patients were categorized in multiple bone and extra-skeletal uptake group (A), 12 in multiple bone uptake without extra-skeletal uptake group (B), 3 in single bone and extra-skeletal uptake group (C) and 8 in single bone uptake without extra-skeletal uptake group (D). In group A, 42/44 patients had metastatic bone disease; where primary lesion was correlating extra-skeletal uptake in $40(91.0 \%)$ cases. Among the 12 patients of group B, $5(41.7 \%)$ had primary malignant bone disease, 3 (25.0\%) had benign bone lesions and $4(33.3 \%)$ had metastatic bone disease but PET-CT failed to indicate the primary site. In group $\mathrm{C}$, PET-CT positive extra-skeletal uptake sites were confirmed as primary lesions in all the 3 patients. Among the 8 patients of group D, $5(62.5 \%)$ were benign bone disease, one (12.5\%) was high grade sarcoma and the remaining two $(25.0 \%)$ were metastatic bone disease where PET-CT failed to identify primary site. Additionally, there was significant $(\mathrm{P}=$ 0.0003 ) difference in prevalence of biopsy sites performed according to the presence of extra-skeletal uptake in PET-CT.

Conclusion: Clinical impact of PET-CT was different according to groups classified with stepwise manners, which suggested that different strategies should be taken especially when there was not extra-skeletal uptake to optimize management plan. PET-CT also had a significant role in patients with extra-skeletal uptake lesion through guidance of easily approachable biopsy sites.

Key words: Carcinoma of Unknown Primary, Skeletal Metastasis, PET-CT

Bangladesh J. Nucl. Med. Vol. 22 No. 2 July 2019

Doi: https://doi.org/10.3329/bjnm.v22i2.51758

\section{INTRODUCTION}

Distant metastasis is generally thought to be a late episode of disease process and indicative to poor prognosis and short life expectancy. Bone is one of the most frequent sites for distant metastasis of cancer (1, 2). But, bone metastasis has also been reported as initial manifestation of cancer even without a known primary. Approximately $10.8 \%-30.0 \%$ of the patients having metastatic carcinoma from unknown primary may present with skeletal metastasis as an initial detectable lesion (3-5). In patients with suspicious bone lesions without known primary malignancy, it is important to distinguish malignant from benign as well as to find primary focus in patients with high probability of bone metastasis for an optimized treatment.

Fluorine-18 fluorodeoxyglucose positron emission tomography-computed tomography (F-18 FDG PET-CT) has been established as an excellent whole body screening modality in detection of primary focus, staging, treatment planning and monitoring of treatment in different types of metastatic cancer of unknown primary (6-13). However, in addition to potential primary cancer identification, role of PET-CT has also been reported in differential diagnosis by recognizing other causes in bone lesions suspicious for metastasis (14).

In this study, we applied a step-wise approach of PET-CT in suspicious bone metastasis patients through classification of imaging findings based on uptake patterns for differential diagnosis as well as identification of primary site and investigated whether 
PET-CT can help clinician for optimized management plans.

\section{PATIENTS AND METHODS}

\section{Patients}

A total of 94 patients, who underwent PET-CT for suspicious bone metastasis as an abnormal bone lesion radiologically between January 2016 and March 2018, were initially enrolled. Exclusion criteria of our study were: the presence of previous known malignancy $(n=$ 4 ) and inadequate follow-up records $(\mathrm{n}=16)$. So, finally 74 patients were included in this study. These patients did not have any history of receiving chemotherapy or radiotherapy prior to PET-CT. All the 74 patients underwent at least one or more imaging modalities before PET-CT. Radiologically an abnormal bone lesion was found by MRI in 42, CT in 20,bone scan in 2, and more than one imaging modalities in 10 patients. This retrospective study has been approved by our institutional review board and the need for written informed consent was waived.

\section{Imaging acquisition and analyses}

PET-CT was performed using a Discovery ST PET-CT system (GE Medical Systems, Milwaukee, WI, USA) following standard protocol of the institution. All patients fasted for 6 hours before intravenous injection of $5.5 \mathrm{MBq} / \mathrm{kg}$ body weight of F-18 FDG. Blood glucose level did not exceed $7.2 \mathrm{mmol} / \mathrm{L}$ in any patient. A low-dose CT scan was performed for attenuation correction at $50 \mathrm{~min}$ after FDG injection. After the CT scan, a PET scan was performed for the duration of 150 sec per bed position. Data were reconstructed using ordered-subset expectation maximization (OSEM) reconstruction (128X128 matrix, 3.7-mm slice thickness, subset: 21, iterations: 2).

PET-CT evaluations were performed by two experts in nuclear medicine. FDG positivity of suspicious lesion was referred as discernible focal uptake at bones or extra-skeletal tissues in comparison with the background and blood pool activity. However, CT positive lesions with mild or non-focal FDG avidity were also considered as positive, as some cancers show low FDG avidity. Each focal extra-skeletal uptake site was considered as compatible to primary lesion or metastatic lesion according to uptake pattern and site distribution. Extra-skeletal accumulation due to physiological cause and inflammation was considered as insignificant; where uptake pattern was non-focal, symmetrical, linear or tracked along soft tissue boundary (as pleural surface or fascial plane).

\section{Study design}

To allocate stepwise approach we contrived several criteria: FDG positivity of bone lesions, multiplicity of positive bone uptake and presence of extra-skeletal uptake (Fig. 1). Patients $(\mathrm{n}=7)$ with negative bone uptake did not show any extra-skeletal uptake. Hence, no malignancy on bone or extra-skeletal region was noted. So, we applied further two steps on only the 'positive bone uptake' patients. According to the multiplicity of bones having FDG uptake, patients were further categorized as 'single bone uptake' and 'multiple bone uptake'. In the last step, both the single bone uptake and multiple bone uptake patients were further categorized into 'extra-skeletal uptake' and 'no extra-skeletal uptake' groups based on presence of FDG in extra-skeletal tissue. So finally, we got four characterized groups of 67 positive bone uptake patients after the step-wise approach (Fig. 1); multiple bone uptake with extra-skeletal uptake patients (A), multiple bone uptake without extra-skeletal uptake patients (B), single bone uptake with extra-skeletal uptake patients (C) and single bone uptake without extra-skeletal uptake patients (D). We compared follow-up results of each group and also determined the prevalence of primary sites. Follow-up was performed by clinically, histopathology and further imaging modalities. Additionally, we investigated whether PET-CT has clinical role to guide biopsy sites through the comparison of the difference of biopsy site according to presence of extra-skeletal uptake.

\section{Statistical analysis}

Continuous variables were expressed as means \pm standard deviation. A Chi-square test (Fisher's exact test) was used to compare categorical variables to assess the diagnostic impact of PET-CT for recommendation of biopsy site. The results were considered statistically significant if the $P$ 
value was less than 0.05 . SPSS for Windows 20.0 program (SPSS Inc., IBM Company Chicago, IL, USA) was used to conduct statistical analyses.

\section{RESULTS}

The 74 study subjects had a mean age of $67.5 \pm 13.9$ years (range: 10-91 years) and male-female ratio 44:30. On PET-CT, 7 (9.5\%) patients showed no FDG uptake in extra-skeletal area as well as bone. Among the remaining 67 patients, PET-CT demonstrated single bone uptake in $11(14.8 \%)$ and multiple bone uptake in 56 (75.7\%) patients. Again from PET-CT, 47 (63.5\%) patients showed positive extra-skeletal FDG uptake and $27(36.5 \%)$ had no extra-skeletal uptake. Final diagnosis of bone lesions revealed malignant bone disease in 57 of 74 patients; whereas benign bone disease was diagnosed in $17(22.9 \%)$ patients. Furthermore, among the former, $6(8.1 \%)$ patients had primary malignant bone disease and $51(69.0 \%)$ had metastatic bone disease (Table 1).

Table 1: Demographic distribution of study population $[n=74]$

\begin{tabular}{ll}
\hline Variables & $\begin{array}{l}\text { Number of patients } \\
\text { (range or \%) }\end{array}$ \\
\hline $\begin{array}{l}\text { Age (y) mean } \pm \text { SD } \\
\text { Male/Female }\end{array}$ & $67.5 \pm 13.9(10-91)$ \\
F-18 FDG positivity of suspicious bone lesion & $44(59.5) / 30(40.5)$ \\
No uptake & \\
Single bone uptake & $7(9.5)$ \\
Multiple bone uptake & $11(14.8)$ \\
Extra-skeletal uptake on PET-CT & $56(75.7)$ \\
Present & \\
Absent & $47(63.5)$ \\
Final diagnosis of suspicious bone lesion on PET-CT & $27(36.5)$ \\
Benign bone disease & \\
Malignant bone disease (Primary) & $17(22.9)$ \\
Malignant bone disease (Metastatic) & $6(8.1)$ \\
Follow-up methods & $51(69 . .0)$ \\
Histopathology & \\
Imaging & $51(68.9)$ \\
Clinical follow-up & $9(12.2)$ \\
\hline
\end{tabular}

Table 2: Distribution of 67 patients with positive bone lesions in PET-CT according to each group

\begin{tabular}{lllll}
\hline & $\begin{array}{l}\text { Group A } \\
(\mathbf{n = 4 4 )}\end{array}$ & $\begin{array}{l}\text { Group B } \\
(\mathbf{n = 1 2})\end{array}$ & $\begin{array}{l}\text { Group C } \\
(\mathbf{n = 3})\end{array}$ & $\begin{array}{l}\text { Group D } \\
(\mathbf{n}=\mathbf{8})\end{array}$ \\
\hline $\begin{array}{l}\text { Malignant bone disease (Metastatic) } \\
\text { Primary lesion detected by PET-CT }\end{array}$ & $40(91.0 \%)$ & 0 & 3 & 0 \\
MCUPs/Primary lesion not detected & by PET-CT* & $2(4.5 \%)$ & $4(33.3 \%)$ & $02(25.0 \%)$ \\
Malignant bone disease (Primary) & 0 & $5(41.7 \%)$ & 0 & $1(12.5 \%)$ \\
Benign bone disease & $2(4.5 \%)$ & $3(25.0 \%)$ & 0 & $5(62.5 \%)$ \\
\hline
\end{tabular}

Group A. Patients having multiple bone uptake of FDG with extra-skeletal uptake

Group B. Patients having multiple bone uptake of FDG without extra-skeletal uptake

Group C. Patients having single bone uptake of FDG with extra-skeletal uptake

Group D. Patients having single bone uptake of FDG without extra-skeletal uptake

*The primary lesion site remained unknown/primary site was not detected by PET-CT,

but by other imaging modalities and/or by histopathological examination. 


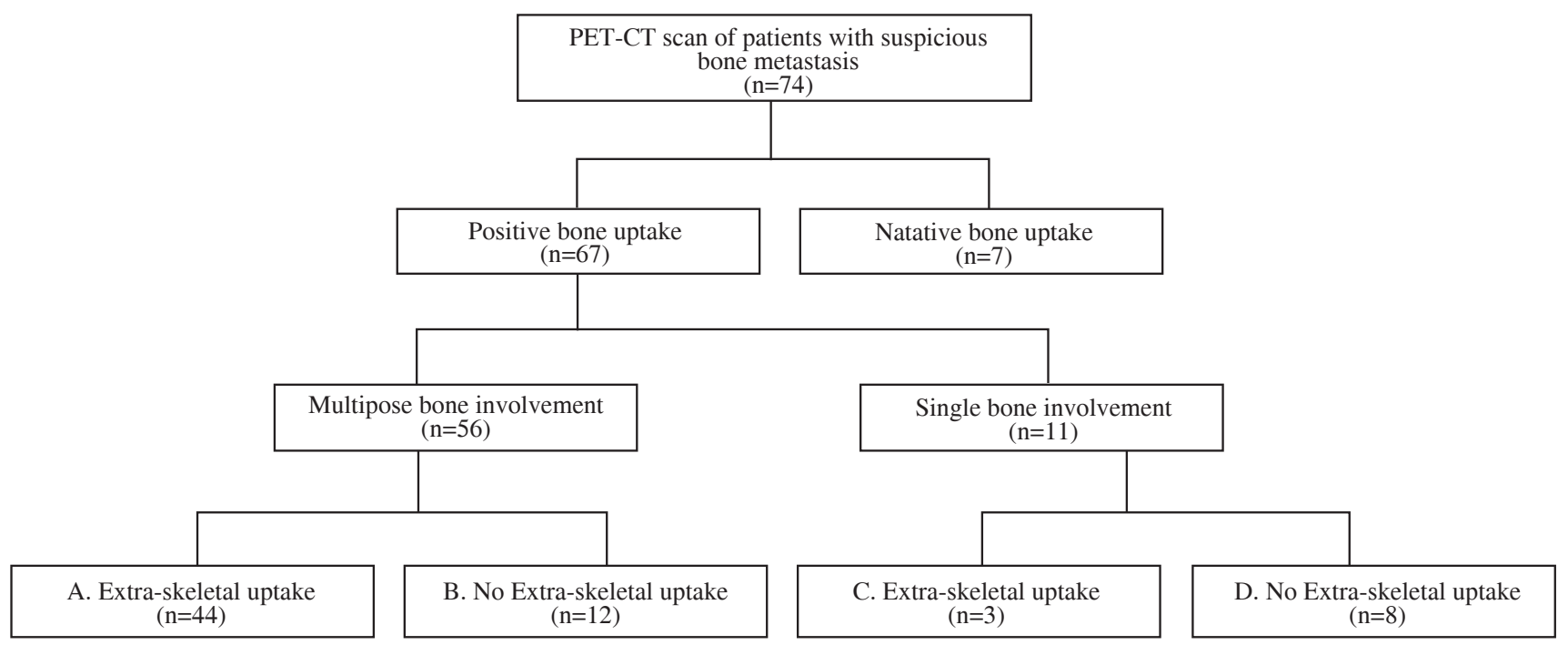

Figure 1: PET-CT aided stepwise diagnostic flow in patients with suspicious bone metastasis from an unknown primary

From stepwise diagnostic flow (Figure 1), 44 patients were categorized in group A, 12 patients in group B, 3 patients in group $\mathrm{C}$ and 8 patients in group D. Table 2 shows the final diagnosis of the FDG positive bone lesions patients $(n=67)$. In group A, extra-skeletal FDG uptake site was confirmed as primary lesion histopathologically in 39 cases and clinically in 1 case. Rest of the 4 patients showed no correspondence of the primary cancer with the positive extra-skeletal FDG uptake sites. In two (4.5\%) patients, bone lesions were finally diagnosed as benign lesion (fractures and inflammatory uptake). These patients also had

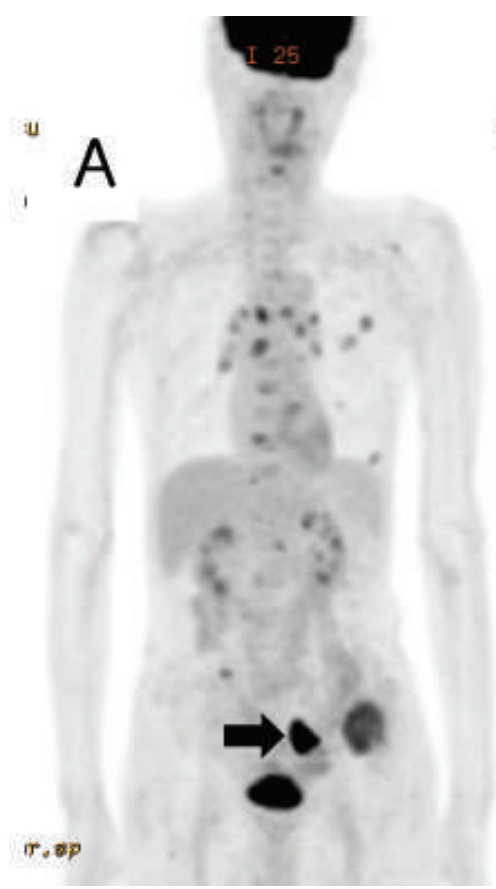

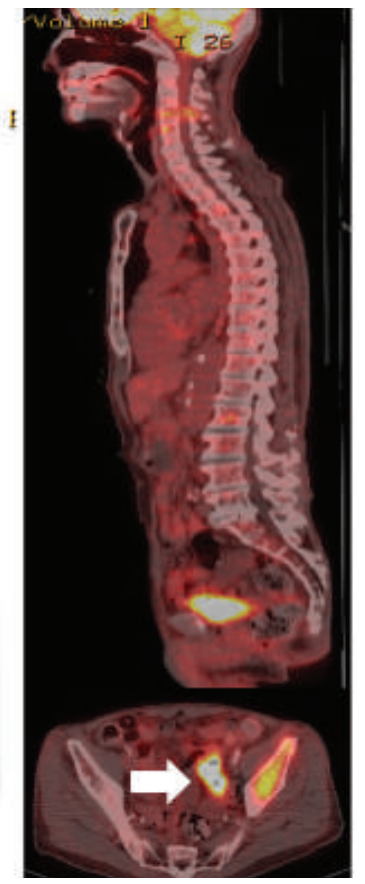

extra-skeletal uptake in stomach and thyroid respectively, which were histopathologically revealed with inflammation. One of the remaining two (4.5\%) patients was confirmed as prostate cancer by further evaluations (histopathology) instead of lung cancer detected by PET-CT. In another patient with metastatic lesions in bone, primary site remained unknown though extra-skeletal uptake was found in multiple lymph nodes of neck, chest, and intra-abdominal regions. Among the 12 patients of group B, 5 (41.7\%) had primary malignant bone disease (multiple myeloma), 3 $(25.0 \%)$ patients had benign (inflammatory) bone

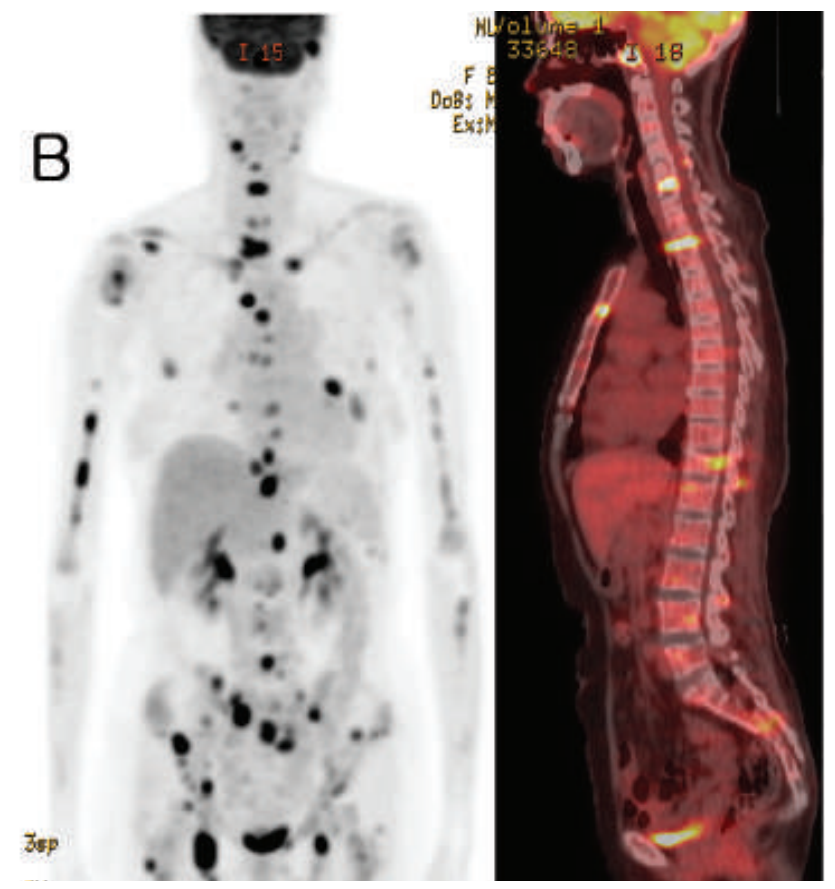



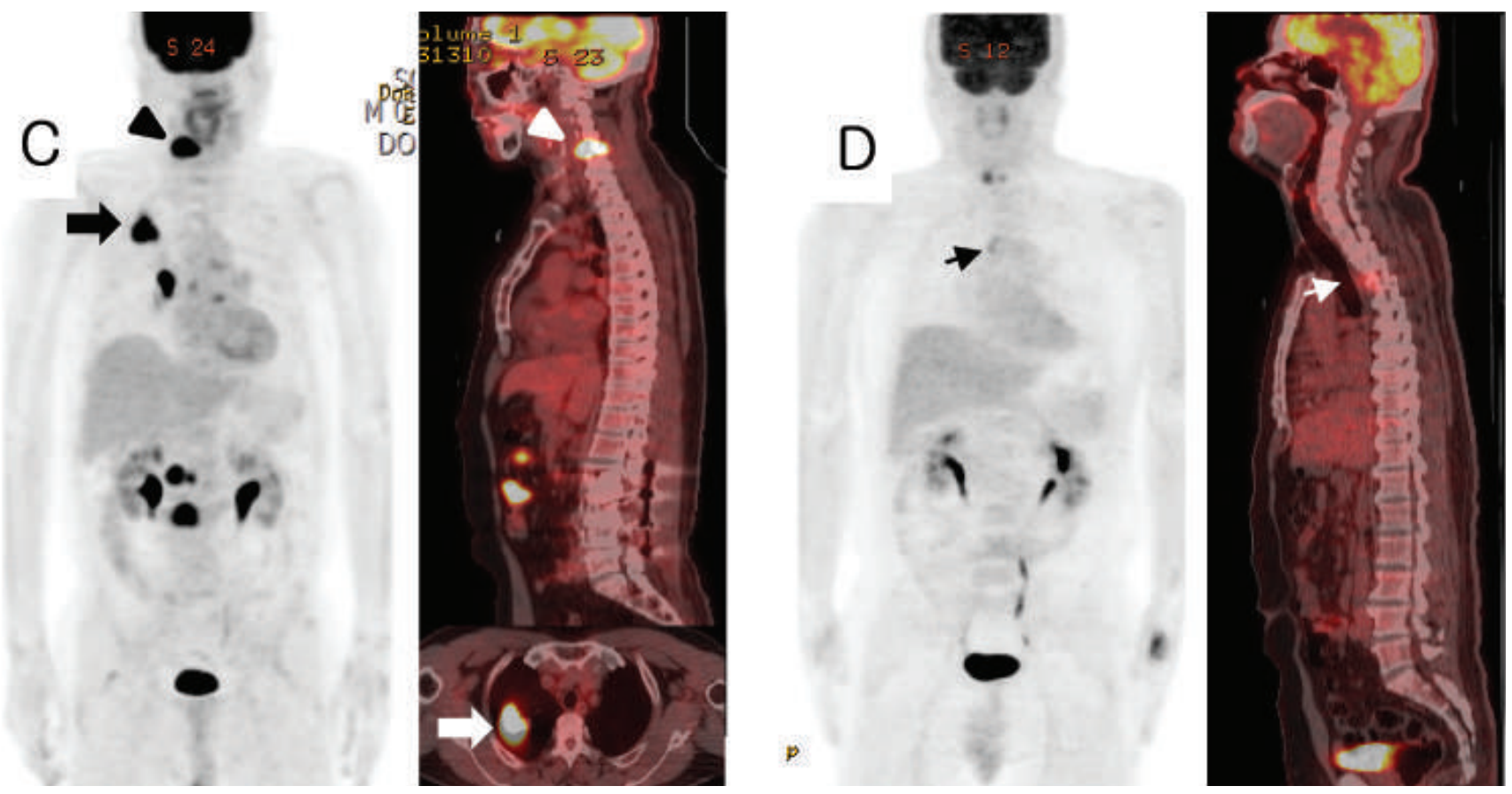

Figure 2: Representative images of each group from F-18 FDG PET-CT

A. A 82-year-old male patient showed multiple hypermetabolic bone lesions. PET-CT also showed hypermetabolic lesion in sigmoid colon (arrow). Histopathological examination confirmed moderately differentiated adenocarcinoma of sigmoid colon.

B. A 61-years-old male patient showed focal hypermetabolic lesions in multiple bones. However, extra-skeletal FDG uptake was not noted. Pathological examination confirmed a case of multiple myeloma.

C. A 75-years-old male patient showed focal hypermetabolic lesion in C5 vertebral body and transverse process (arrowhead). PET-CT also showed hypermetabolic focal lesion in apical segment of right upper lobe (arrow). Histopathological examination confirmed as adenocarcinoma of right lung.

D. A 55-years-old male patient showed single hypermetabolic lesion in T4 vertebral body (arrow). However, extra-skeletal uptake of FDG was not seen. Histopathological examination revealed mixed inflammation with mild marrow fibrosis in the affected bone.

lesions and $4(33.3 \%)$ had metastatic malignant bone disease but PET-CT failed to detect the primary. Primary lesions were confirmed in two of the four cases by further evaluations; one in thyroid and another in splenic flexure of colon. Primary lesion remained unknown in two cases of this group. In case of all the three patients from group C, PET-CT positive extra-skeletal uptake sites were confirmed as primary lesions by histopathology. Among the 8 patients of group D, one $(12.5 \%)$ was finally diagnosed as high grade sarcoma, 5 $(62.5 \%)$ as benign bone disease (two inflammatory lesions, one hemangioma, one tuberculosis and one fracture) and the remaining two $(25.0 \%)$ as metastatic bone disease; where primary lesion site was prostate in both cases, not identified by PET-CT but confirmed by further evaluation (histopathology). Representative cases of each groups are illustrated in Figure 2.

PET-CT correctly identified primary site in a total of 43 (40 from group A and 3 from group C) of 51 patients with metastatic bone disease (Table 2). The most frequent primary site indicated by PET-CT was lung, accounting 19 (44.3\%) cases, followed by $9(21.0 \%)$ prostate, $4(9.3 \%)$ liver and hepatobiliary, $4(9.3 \%)$ thyroid, 3 (6.9\%) stomach, 2 (4.6\%) colon, $1(2.3 \%)$ pancreas and $1(2.3 \%)$ kidney. PET-CT was unable to identify primary site in 8 patients but primary site could be found in 5 by other modalities, where prostate was the most frequent (3 (60\%) cases) site, followed by 1 $(20.0 \%)$ thyroid and $1(20.0 \%)$ colon (Table 3$)$. 
Table 3: Prevalence of primary sites in patients with suspicious bone metastasis $(n=48)$

\begin{tabular}{lcc}
\hline Primary sites & $\begin{array}{c}\text { Detected by } \\
\text { PET-CT }(\mathbf{n}=43)\end{array}$ & $\begin{array}{c}\text { Not detected by } \\
\text { PET-CT (n= 5) }\end{array}$ \\
\hline Lung & $19(44.3 \%)$ & 0 \\
Prostate & $9(21.0 \%)$ & $3(60 \%)$ \\
Liver and hepatobiliary & $4(9.3 \%)$ & 0 \\
Thyroid & $4(9.3 \%)$ & $1(20 \%)$ \\
Stomach & $3(6.9 \%)$ & 0 \\
Colon & $2(4.6 \%)$ & $1(20 \%)$ \\
Pancreas & $1(2.3 \%)$ & 0 \\
Kidney & $1(2.3 \%)$ & 0 \\
\hline
\end{tabular}

To see the diagnostic impact of PET-CT for recommendation of biopsy site, only patients with positive bone uptake who underwent biopsy after PET-CT (39 cases from group A, 2 from group B, 3 from group C and 2 from group D), was further evaluated (Table 4). Among 46 patients, 42 patients who had presence of extra-skeletal lesion on PET-CT underwent tissue biopsy in sites solely recommended by PET-CT. On the other hand, in negative extra-skeletal uptake group, biopsy was done at the site recommended by colonoscopy in one patient and suspicious bones localized in both PET-CT and other conventional imaging in 3 patients. Fisher's exact test showed PET-CT significantly $(\mathrm{P}=0.0003)$ had an impact on recommendation for biopsy site in these patients. Furthermore, among the 42 patients with presence of extra-skeletal lesion, biopsy was performed from primary lesion site in 35 cases and additional superficial extra-skeletal metastatic sites (scalp in 1 and lymph nodes in 6 patients) in 7 patients indicated by PET-CT. It suggested that PET-CT was very useful to find easily approachable biopsy site.

To see the diagnostic impact of PET-CT for recommendation of biopsy site, only patients with positive bone uptake who underwent biopsy after PET-CT (39 cases from group A, 2 from group B, 3 from group C and 2 from group D), was further evaluated (Table 4). Among 46 patients, 42 patients who had presence of extra-skeletal lesion on PET-CT underwent tissue biopsy in sites solely recommended by PET-CT. On the other hand, in negative extra-skeletal uptake group, biopsy was done at the site recommended by colonoscopy in one patient and suspicious bones localized in both PET-CT and other conventional imaging in 3 patients. Fisher's exact test showed PET-CT significantly $(\mathrm{P}=0.0003)$ had an impact on recommendation for biopsy site in these patients. Furthermore, among the 42 patients with presence of extra-skeletal lesion, biopsy was performed from primary lesion site in 35 cases and additional superficial extra-skeletal metastatic sites (scalp in 1 and lymph nodes in 6 patients) in 7 patients indicated by PET-CT. It suggested that PET-CT was very useful to find easily approachable biopsy site.

Table 4: Diagnostic impact of PET-CT for recommendation of biopsy site according to extra-skeletal uptake positivity

\begin{tabular}{lcccc}
\hline & $\begin{array}{c}\text { Biopsy from } \\
\text { sites other } \\
\text { than bones }\end{array}$ & $\begin{array}{c}\text { Biopsy } \\
\text { from bone } \\
\text { lesions }\end{array}$ & Total P value \\
\hline $\begin{array}{l}\text { Presence of extra-skeletal } \\
\text { lesion on PET-CT }\end{array}$ & $42 *$ & 0 & 42 & \\
$\begin{array}{l}\text { Absence of extra-skeletal } \\
\text { lesion on PET-CT }\end{array}$ & $1 \uparrow$ & $3 \uparrow$ & 4 & 0.0003 \\
\hline Total & $\mathbf{4 3}$ & $\mathbf{3}$ & $\mathbf{4 6}$ \\
\hline
\end{tabular}

*Biopsy site recommended by PET-CT.

$\dagger$ Biopsy site recommended by other modality.

$\$$ Biopsy was performed by localization of the same sites in conventional modalities and/or PET-CT.

\section{DISCUSSION}

Carcinoma of unknown primary is a condition characterized as an early dissemination of metastasis in which no primary site can be detected with the patient history, physical examination, extensive laboratory testing and wide range of radiological investigations (15, 16). The incidence of metastasis without identification of primary cancer has been reported as 3\%-5\% and approximately $10.8 \%-30 \%$ of these patients have skeletal metastasis as presenting symptoms $(3-5,15)$. In patients of skeletal metastasis with unknown primary, the diagnosis and accurate managements are often delayed in spite of extensive imaging procedures and pathological investigations, which exert negative effect on prognosis through skeletal related events such as the risk of fractures, hypercalcemia, and spinal cord compression (2). It has been suggested that appropriate detection of a 
primary tumor can make it possible to optimize treatment planning and to improve patient outcome $(17,18)$.

There are a wide spectrum of diagnostic procedures including various laboratory tests, radiological, endoscopic examinations, serum tumor marker studies, and histological diagnosis for primary tumor detection. However, these procedures can be expensive, time-consuming and invasive and eventually may fail to detect primary tumor (15). PET-CT has an established role in patients with metastasis of an unknown primary tumor because of its ability to screen whole body in a single set-up. A recent meta-analysis showed an overall $40.93 \%$ primary site detection rate with high sensitivity and specificity by application of PET-CT in patients of metastasis with unknown primary (6). Another group of researchers showed that PET-CT is useful not only for detection of primary site but also for characterization of abnormal bone lesion (14). But, previous study only showed the correlation between FDG avidity of suspicious bone lesions and identification of primary focus by PET-CT, which had limitations to apply PET-CT findings to clinical practice in those patients in the aspect of recommendation of the next management plan.

We tried to classify suspicious bone lesions into groups with step-wise manner based on PET-CT findings that enable clinicians to consider different management strategies in each group. When patients had both bone and extra-skeletal uptakes in PET-CT (group A and C), extra-skeletal uptake sites were finally identified as primary lesion in most of the patients. Interestingly, PET-CT showed maximum primary site detection rate in patients with single bone uptake. Such performance can make clinicians continue to evaluate according to PET-CT results in these groups. On the other hands, a wide range of differential diagnosis was necessary in patients only with multiple bone uptakes (group B). Especially in $33.3 \%$ case of metastatic bone disease, where primary foci were not identified by PET-CT but other modalities such as colonoscopy and neck ultrasound. In patients who only had single bone uptake (group D), metastatic bone disease (25\%) and primary malignant bone tumor $(12.5 \%)$ were diagnosed although major proportion of patients $(62.5 \%)$ had benign bone disease as follow-up results. Therefore, a clinician had to investigate further by using tumor markers, other imaging modalities or endoscopic examination considering clinical symptoms, signs as well as PET-CT findings in these groups (B and D).

However, primary site was recognized by further investigations in $5 / 8$ patients that PET-CT failed to identify. Among the undetected primary sites by PET-CT, prostate was the most frequent ( $3 / 5$ patients). The slow rate of glycolysis of tumor cells and low FDG avidity, which could be one of the reason of low detectability by PET-CT (19).Cancer tissue in prostate might be also masked by physiological FDG accumulation in urinary bladder(20).However, increased cell proliferation and accelerated glucose metabolism in metastatic lesion over primary lesion has also been demonstrated as the cause of higher sensitivity of FDG PET-CT for detection metastatic lesions than primary focus of prostate (19).

On the other hand, the second most primary site identified by PET-CT was also prostate $(21.0 \%)$ next to lung $(44.3 \%)$ in our study. In previous studies prostate cancer was observed with high prevalence next to lung cancer or multiple myeloma among patients who had skeletal metastasis as initial manifestation with unknown primary cancer $(4,5)$. Prostate cancer is often confined to prostate tissue, which remain asymptomatic for long time. However, bone pain, other clinical symptoms or sign such as abnormal imaging findings and high level of prostate specific antigen (PSA) can be generated when they metastasized to bones (21).

Pathologic diagnosis through tissue biopsy is very important because clinicians should consider this result to choose therapeutic agents. Especially, mutation profiles as well as histological characteristics have been crucial to apply targeted or immune-therapy (22).In bone metastasis patients, clinicians usually wanted to find other tissues or organs than bone because limited accessibility and painful procedure of bone biopsy can often fail to provide reliable result especially in small sized lesion (23). In our study, we observed significant difference in selection of biopsy site according to presence of extra-skeletal uptake in PET-CT (Table 4). Furthermore, biopsy was performed in other extra-skeletal uptake sites which were superficial or easily accessible, such as lymph nodes or scalp, than primary focus in 7/42 (16.7\%) sites. It suggests PET-CT can provide a valuable role by localization of extra-skeletal uptake site which can be easily approachable for biopsy. 
There are several limitations in our study. Firstly, our study is designed in retrospective manner. So, this study has case selection bias affecting the representativeness and reliability. Secondly, all bone lesions were not confirmed by histopathology especially in benign bone diseases group. Thirdly, we could not use other imaging modalities and tumor marker study in diagnosis process especially in patients without extra-skeletal uptake. Further studies are warranted through adding those parameters to improve diagnostic performance of PET-CT.

\section{CONCLUSION}

Clinical impact of PET-CT was different according to groups classified with stepwise manners, which suggested that different strategies should be taken especially when there was not extra-skeletal uptake to optimize management plan. PET-CT also had a significant role in patients with extra-skeletal uptake lesion through guidance of easily approachable biopsy sites.

\section{Acknowledgement}

We acknowledge the contribution of Dr. Sae-Ryung Kang, Dr. Su Woong Yoo of Department of Nuclear Medicine, Chonnam National University Hwasun Hospital in image analysis and data collection.

This study was previously published as an interesting image in Clinical Nuclear Medicine, September 2019 Volume 44 - Issue 9 - p e 524-e525

\section{REFERENCES}

1. Rougraff BT. Evaluation of the patient with carcinoma of unknown origin metastatic to bone. Clin Orthop Relat Res. 2003(415 Suppl):S105-9.

2. Coleman RE. Skeletal complications of malignancy. Cancer. 1997;80(8 Suppl):1588-94.

3. Nottebaert M, Exner GU, von Hochstetter AR, Schreiber A. Metastatic bone disease from occult carcinoma: a profile. Int Orthop. 1989;13(2):119-23.

4. Katagiri H, Takahashi $\mathrm{M}$, Inagaki J, Sugiura $\mathrm{H}$, Ito $\mathrm{S}$, Iwata $\mathrm{H}$. Determining the site of the primary cancer in patients with skeletal metastasis of unknown origin: a retrospective study. Cancer. 1999;86(3):533-7.

5. Takagi T, Katagiri H, Kim Y, Suehara Y, Kubota D, Akaike K, et al. Skeletal Metastasis of Unknown Primary Origin at the Initial Visit: A Retrospective Analysis of 286 Cases. PLoS One. 2015;10(6):e0129428.

6. Burglin SA, Hess S, Hoilund-Carlsen PF, Gerke O. 18F-FDG PET/CT for detection of the primary tumor in adults with extracervical metastases from cancer of unknown primary: A systematic review and meta-analysis. Medicine. 2017;96(16):e6713.

7. Kwee TC, Basu S, Cheng G, Alavi A. FDG PET/CT in carcinoma of unknown primary. Eur J Nucl Med Mol Imaging. 2010;37(3):635-44.
8. Surucu E AM, Şengöz T, Demir Y, Durak H. . The value of F-18 FDG PET-CT for detecting primary foci in the metastatic cancer of unknown primary origin. Eastern Journal of Medicine. 2014;19(2):79-83.

9. Elboga U, Kervancioglu S, Sahin E, Basibuyuk M, Celen YZ, Aktolun C. Utility of F-18 fluorodeoxyglucose positron emission tomography/ computed in carcinoma of unknown primary. Int J Clin Exp Pathol. 2014;7(12):8941-6.

10. Bakhshayeshkaram M GM, Hassanzad M, Doroudinia A, Jamaati H, Aghahosseini F. Diagnostic performance of F-18 FDG PET/CT in patients with cancer of unknown primary: additional benefit over CT-based conventional work up. Novelty in Biomedicine. 2016;4(1)(Jan 18):5-12.

11. Lee JR, Kim JS, Roh JL, Lee JH, Baek JH, Cho KJ, et al. Detection of occult primary tumors in patients with cervical metastases of unknown primary tumors: comparison of (18)F FDG PET/CT with contrast-enhanced CT or CT/MR imaging-prospective study. Radiology. 2015;274(3):764-71.

12. Fan HB, Wang AJ, Yang DL, Xiao J, Ai Y, Huang L, et al. Use of (18)F-FDG PET/CT to locate primary malignancies in patients with hepatic cirrhosis and malignant ascites. Chinese journal of cancer research = Chung-kuo yen cheng yen chiu. 2013;25(5):500-4.

13. Koc ZP, Kara PO, Dagtekin A. Detection of unknown primary tumor in patients presented with brain metastasis by F-18 fluorodeoxyglucose positron emission tomography/computed tomography. CNS Oncol. 2018;7(2):CNS12.

14. Park SB, Park JM, Moon SH, Cho YS, Sun JM, Kim BT, et al. Role of 18F-FDG PET/CT in patients without known primary malignancy with skeletal lesions suspicious for cancer metastasis. PLoS One. 2018;13(5):e0196808.

15. Pavlidis N, Khaled H, Gaafar R. A mini review on cancer of unknown primary site: A clinical puzzle for the oncologists. J Adv Res. 2015;6(3):375-82.

16. Abbruzzese JL, Abbruzzese MC, Lenzi R, Hess KR, Raber MN. Analysis of a diagnostic strategy for patients with suspected tumors of unknown origin. Journal of clinical oncology : official journal of the American Society of Clinical Oncology. 1995;13(8):2094-103.

17. Choi J, Nahm JH, Kim SK. Prognostic clinicopathologic factors in carcinoma of unknown primary origin: a study of 106 consecutive cases. Oncotarget. 2017;8(37):62630-40.

18. Haas I, Hoffmann TK, Engers R, Ganzer U. Diagnostic strategies in cervical carcinoma of an unknown primary (CUP). Eur Arch Otorhinolaryngol. 2002;259(6):325-33.

19. Liu Y. Diagnostic role of fluorodeoxyglucose positron emission tomography-computed tomography in prostate cancer. Oncol Lett. 2014;7(6):2013-8.

20. Shreve PD, Grossman HB, Gross MD, Wahl RL. Metastatic prostate cancer: initial findings of PET with 2-deoxy-2-[F-18]fluoro-D-glucose. Radiology. 1996; 199(3): 751-6.

21. Kirakoya B, Hounnasso PP, Pare AK, Mustapha AB, Zango B. Clinico-Pathological Features of Prostate Cancer at the University Hospital Yalgado Ouedraogo, Ouagadougou, Burkina Faso. J West Afr Coll Surg. 2014;4(4):70-81.

22. Ofiara LM, Navasakulpong A, Ezer N, Gonzalez AV. The importance of a satisfactory biopsy for the diagnosis of lung cancer in the era of personalized treatment. Curr Oncol. 2012;19(Suppl 1):S16-23.

23. Adams HJ, Nievelstein RA, Kwee TC. Opportunities and limitations of bone marrow biopsy and bone marrow FDG-PET in lymphoma. Blood Rev. 2015;29(6):417-25. 